\title{
Physical Function, Psychological Adjustment, and Self-Efficacy Following Sudden Cardiac Arrest and an Initial Implantable Cardioverter Defibrillator (ICD) in a Social Cognitive Theory Intervention: Secondary Analysis of a Randomized Control Trial
}

Cynthia M. Dougherty ( $\square$ cindyd@uw.edu )

University of Washington

Ana Carolina Sauer Liberato

Evidera PPD

Megan M. Streur

University of Washington

Robert L. Burr

University of Washington

Judy Ka Yee Kwan

University of Washington

Tao Zheng

University of Washington

Jon P. Auld

University of Washington

Elaine A. Thompson

University of Washington

\section{Research Article}

Keywords: sudden cardiac arrest, intervention, quality of life, physical function, symptoms, psychological, social cognitive theory, implantable cardioverter defibrillator

Posted Date: January 31st, 2022

DOI: https://doi.org/10.21203/rs.3.rs-1182256/v1

License: @ (i) This work is licensed under a Creative Commons Attribution 4.0 International License. Read Full License 


\section{Abstract}

Background Sudden cardiac arrest (SCA) survivorship results in unique issues in return to physical and psychological function. The purpose of the study was to compare recovery across the first year between SCA survivors and other arrhythmia patients who received an initial implantable cardioverter defibrillator (ICD) for secondary prevention, participating in a social cognitive theory (SCT) intervention.

Methods 168 (129 males, 39 females) who received an ICD for secondary prevention (SCA N=65; other arrhythmia $\mathrm{N}=103)$ were randomized to one of two study conditions: SCT intervention $(\mathrm{N}=85)$ or usual care $(\mathrm{N}=83)$. Outcomes were measured at baseline hospital discharge, 1, 3, 6, \& 12 months: 1) Physical Function: Patient Concerns Assessment (PCA), SF-36 (PCS); 2) Psychological Adjustment: State Trait Anxiety (STAI), CES-D depression, SF-36 (MCS); 3) Self-Efficacy: Self-Efficacy (SCA-SE), Self-management Behaviors (SMB), Outcome Expectations (OE). Outcomes were compared over 12 months for intervention condition x ICD indication using general estimating equations.

Results Participants were Caucasian (89\%),age 63.95+12.3 years, mean EF\% 33.95+13.9, BMI 28.19+6.2, and Charlson Index 4.27+2.3. Physical symptoms (PCA) were higher over time for SCA survivors compared to the other arrhythmia group $(p=0.04)$, ICD shocks were lower in SCA survivors in the SCT intervention $(p=0.01)$; psychological adjustment (MCS) was significantly lower in SCA survivors in the SCT intervention over 6 months, that improved at 12 months $(\mathrm{p}=0.05)$; outcome expectations $(\mathrm{OE})$ were significantly lower for SCA survivors in the SCT intervention $(p=0.008)$.

Conclusions SCA survivors had greater number of physical symptoms, lower levels of mental health and outcome expectations over 12 months despite participation in a SCT intervention.

Trial registration: Clinicaltrials.gov: NCT04462887

\section{Background}

Sudden cardiac arrest (SCA) is the cessation of cardiac mechanical activity that occurs without warning or with symptoms of $<1$ hours duration [1]. SCA results from multiple cardiac causes such as structural heart disease, coronary artery disease, and heart failure. Age-adjusted mortality rates for SCA in the U.S. have declined from 138/100,000 in 1999 to $97.1 / 100,000$ in 2017, thus impacting about 180,202 adults/year [2]. In 2018, survival to discharge following out-of-hospital SCA in US adults was $10.4 \%$, with a range of $7.8 \%$ (DC)-15.3\% (WA state). Survival following in-hospital cardiac arrest is reported at $25.8 \%$ during the same time period. In patients with out-of-hospital cardiac arrest, acute coronary syndrome and other cardiac disease account for the largest proportion of causes, while the most predominant cause of in-hospital SCA is respiratory failure [3]. Approximately 70,000 individuals are discharged from the hospital each year after combined out-of-hospital and in-hospital cardiac arrest [4]. In the post-resuscitation period, the majority of survivors of SCA with cardiac disease will receive an implantable cardioverter defibrillator (ICD) as the mainstay of treatment.

At the time this study was conducted, criteria for receiving an ICD for the secondary prevention of SCA included: 1) survivors of ventricular fibrillation (VF) or hemodynamically unstable ventricular tachycardia (VT), 
2) structural heart disease with spontaneous sustained VT, 3) syncope with sustained VT or VF induced with electrophysiological study (EPS), or 4) non-sustained VT due to prior myocardial infarction (MI), left ventricular ejection fraction (LVEF) $\% \leq 40 \%$, and inducible VF or sustained VT at EPS [5]. Survivors of SCA and those who receive an ICD are a heterogenous group, ranging from those who have no known cardiac disease to those who have complicated chronic cardiac conditions. However, SCA survivors present with unique cognitive, psychological, emotional, and functional needs as a consequence of resuscitation [6]. These unique needs can prevent return to work and normal daily activities, as well as performing family and social roles impacting overall quality of life. As well, evidence suggests that consequences of SCA survival can exist for long periods of time [1]. Interventions have been tested to address these unique needs and promote postSCA adjustment $[10,11]$, but a consistent structured and comprehensive approach to assessment and rehabilitation after SCA is not available or part of routine post-arrest care.

Within the secondary prevention ICD group, comparisons of physical and mental health in SCA survivors vs. others who have complex cardiac arrhythmias but have not suffered SCA, are rarely reported. Herrmann et al [7] demonstrated that QOL (general performance, social capacity, positive mood, social-emotional well-being, cardiac symptom load) was similar in patients with an ICD at 1.5 years after implant compared to those with CAD, but without an ICD. The exception was in those that received ICD shocks, who demonstrated higher psychological distress and lower QOL. Arteaga et al [8] demonstrated that in survivors of SCA who either received an ICD $(\mathrm{N}=45)$, amiodarone $(\mathrm{N}=30)$, or those with CAD and no arrhythmias $(\mathrm{N}=29)$, QOL was higher in those with an ICD or amiodarone compared to those with CAD, and there was no difference in QOL between those with an ICD or taking amiodarone. In the Amiodarone vs. Implantable Defibrillator (AVID) trial [9], physical and mental health scores were similar at baseline and during 12 months of follow-up in both groups of secondary prevention ICD patients. Thus, in those that get a secondary prevention ICD or anti-arrhythmic medication, both groups have similar QOL but comparisons by SCA or other arrhythmia groups are not available.

The purpose of this study was to describe longitudinal change in physical and mental health over the first year post-implant for two ICD secondary prevention groups: patients who experienced a sudden cardiac arrest (SCA) and those who received an ICD for other complex cardiac arrhythmias. We hypothesized that intervention effects across time would be moderated by a history of SCA [intervention x time x ICD indication]. This study fills a major gap in the scientific literature by identifying potential differences between SCA survivors and other ICD secondary prevention patients in response to an intervention program, designed to enhance quality of life and return to function. This study provides findings to address specific needs and experiences of SCA survivors, and describes outcomes comparable to the general ICD population.

\section{Methods}

\section{Design}

This secondary analysis is based on a longitudinal randomized clinical trial that tested the effects of a 2month, combined education and telephone intervention, delivered by trained cardiovascular nurses, compared to usual care (UC) $[10,11]$. Measurements were collected the first week after hospital discharge, and at 1, 3, 6 and 12 months following hospitalization for the initial ICD implant. All research procedures were reviewed and 
approved by hospital Institutional Review Boards (IRBs) and the academic IRB prior to contact with potential participants. The protocol was performed in accordance with relevant guidelines and regulations. Participants were identified during hospitalization by catheter laboratory nurses who worked in medical centers in the Pacific Northwest. Patients who were interested in the study were contacted by telephone the day after hospital discharge by the investigators who explained the study and obtained verbal consent to participate. Then, written informed consent and baseline measures were completed and participants were randomized to study condition (SCT intervention vs UC) using a random number generator program.

The sample included patients who received an initial ICD for secondary prevention. In this sample of 168,65 patients had suffered an out-of-hospital cardiac arrest, achieved return of spontaneous circulation (ROSC), and were admitted and discharged from the hospital alive. The other 103 patients received an ICD for other secondary prevention reasons, including symptomatic and asymptomatic ventricular arrhythmias, with or without electrophysiologic (EPS) testing. The original RCT was conducted between 1999-2003, a time that preceded implantation of ICDs for primary prevention of SCA. During this trial, the use of therapeutic hypothermia was not a standard of care for out-of-hospital cardiac arrest. All patients in this study received implantation of a transvenous lead ICD system. This analysis reports comparisons between the SCA and the other cardiac arrhythmia group by intervention condition over a 12-month period.

\section{Sample}

Study participants $(\mathrm{n}=168)$ included individuals who had experienced a first out-of-hospital SCA or lifethreatening ventricular arrhythmia requiring ICD implantation based on established guidelines [12]. None of the study participants received an ICD for primary prevention reasons. Criteria also included the ability to read, speak and write English, having telephone access, and willingness to be followed for 1 year. Individuals were excluded from the study if they had significant clinical comorbidities that prevented their return home after hospitalization or if they were younger than 21 years of age. Confirmation of SCA and the need for ICD implantation were verified using medical records and EPS reports. All participants were screened at recruitment using the Short Blessed cognitive screening tool [13]. Short Blessed scores $\geq 10$ indicated cognitive impairment too severe for participation. Two individuals were not eligible for study participation based on the Short Blessed criteria.

\section{Social Cognitive Theory (SCT) Intervention}

The SCT intervention consisted of two key components: (1) structured information (SI) provided in a booklet mailed to study participants, and (2) nursing telephone support (NTS) conducted by expert cardiovascular nurses. Expert cardiovascular nurses were had a minimum of 5 years of experience as a cardiovascular nurse and had extensive training in the NTS protocol. Intervention nurses were part of the research team, not employed as staff nurses in the participating medical centers, and did not deliver usual care.

The intervention components were based on Bandura's SCT [14] and previous research with survivors of SCA [15]. A complete description of the theoretical framework used [16] and the nursing intervention is published [17]. The nursing intervention was unlike any interventions currently used in clinical practice and was specifically designed to match the Domains of Concern previously validated with this population. The SI booklet, Sudden Cardiac Arrest: A Survivor's Experience, contains two components: (1) a descriptive 
component including individual verbatim statements about experiences of others during the first year of recovery and (2) a management component outlining successful strategies (skills) used by others in dealing with issues in recovery. The purpose of the SI booklet was to describe the experiences one can expect to encounter during recovery after an ICD and offer suggestions for behavioral strategies to deal with the issues.

The NTS protocol was a telephone intervention delivered over 2 months subsequent to ICD implantation. The purposes of the NTS intervention were to (1) teach specific knowledge and behavioral skills needed to manage ICD recovery, (2) enhance self-confidence (self-efficacy) in one's ability to deal with illness demands, and (3) re-duce emotional arousal and anxiety. Telephone calls were designed to last approximately 15-20 minutes. Each call was carefully scripted to include the following elements: (1) check-in about current concerns, (2) assessment of the topic for the week, (3) review of common recovery experiences, (4) discussion of behavioral strategies for dealing with the topic for the week, (5) provision of positive feedback for strategies already working well, (6) anxiety reduction statements, (7) practice of new behaviors using role-playing and problem solving techniques, (8) summarization, (9) setting specific goals for the upcoming week, and (10) collaborating on a learning assignment for the sub-sequent week. Content of the nursing intervention included physical symptoms, activity progression and exercise, emotional reactions, ICD shocks, partner relationships, safety and maintenance of the ICD, and dealing with health care providers.

Intervention participants received study materials following completion of the baseline questionnaire. Participants were asked to read the SI booklet within the first week after hospital discharge and to refer to it during NTS calls. All intervention calls occurred during the first 2 months following ICD implantation. In addition to the NTS calls, intervention participants could access the intervention nurse during regular business hours via a toll free telephone Monday to Friday, or via pager after hours (24 hours/day). Over a 2-year period, $16(19 \%)$ of 84 individuals used the nurse pager a total of 18 times, the majority of whom called about the ICD (75\%). The other $25 \%$ of calls were related to general medical topics.

Usual care participants received standard treatment from their health care providers. Usual care consisted of standardized hospital-based education about the ICD in the form of a booklet, videotape, or both. A pre-study program review revealed that the type and content of information provided during hospitalization were similar across the ten recruitment sites. Both groups were monitored for exposure to additional interventions, participation in educational or support groups, and counseling. In both groups, $97 \%$ reported receiving ICD device information and/or viewing an ICD related videotape during their hospitalization for ICD implantation. Both groups received outpatient follow-up clinic visits at times and frequency as designated by their health care providers.

\section{Measures}

Study outcomes were measured 5 times over 12 months, starting with hospital discharge following ICD implant and then at 1, 3, 6, 12 months later. Unless otherwise noted, higher values denote higher levels of the measured construct; reported internal consistency values (Cronbach's alpha, a) are based on the study sample. 


\section{Outcome $\quad$ Description of Measure
Variable \& \\ Measures}

\section{Physical \\ Function}

Patient

Concerns

Assessment

(PCA) [18]

\section{Short Form}

Health Survey

(SF-12) [19]

SF-12 is a 12-item measure of general health related to physical and mental health. Two component summary scales of physical (PCS, $a=0.94$ ) and mental health (MCS, $a=$ $0.94)$ are derived.

Cardiac arrhythmias

\section{Psychological Adjustment}

Anxiety [20]

State-Trait Anxiety Inventory (STAI) measure of anxiety $(a=0.84)$ is used extensively in cardiovascular populations. Higher scores denote higher anxiety. This study used the state scale of the inventory only.

Depression [21]
ICD shocks and heart rhythm stability assessed using interrogation reports from ICD devices during routine follow-up visits. The total number of ICD shocks received whether or not appropriate for the cardiac rhythm were recorded.
Center for Epidemiologic Studies Depression (CES-D $)^{16}$ is a measure $(a=0.87)$ of depressive symptomatology in the general population with an emphasis on depressed

\section{Self-Efficacy}

Self-Efficacy Sudden cardiac arrest-self efficacy (SCA-SE) scale focuses on self-efficacy expectations Expectations [22] mood.

\section{Self-Efficacy Behaviors [22]}

\section{Outcome} Expectations [22] about one's ability to manage common problems after sudden cardiac arrest and an ICD $(a=0.93)$.

Sudden cardiac arrest behaviors (SCA-B) scale focuses on behaviors required to manage common problems after sudden cardiac arrest and an ICD $(a=0.89)$.

Sudden cardiac arrest outcome expectations (SCA-OE) $(\alpha=0.81)$ focus on perceived consequences of engaging in self-management behaviors after sudden cardiac arrest and ICD receipt.

\section{Analysis}

Initially, the distributional properties of the study variables, outlier cases, and missing data were characterized. As the proportion of missing data was less than $5 \%$, imputation procedures were not used [23]. Descriptive statistics (mean, SD) and histogram displays were used to compare sociodemographic variables for the full sample, and the four independent groups defined by ICD indication and intervention condition. Oneway ANOVA was used (SPSS version 19.0) to describe baseline differences among four groups defined by (1) SCT Intervention or usual care) and (2) ICD indication: sudden cardiac arrest or other cardiac arrhythmia. The effects of ICD indication on intervention outcomes were compared using a two-factor, repeated measures 
design with generalized estimating equations (GEE), comparing changes from baseline to 12 months for indicators of physical function, psychological adjustment, and self-efficacy ( $p \leq 0.05)$. The effect of ICD indication on intervention outcomes was examined across the 12-months post-ICD (Table 2), adjusting for the baseline values of outcome measures as well as age, gender, and ethnicity. These analyses were exploratory, designed to uncover potential differences associated with SCA, thus no adjustments were made for multiple tests [24]. Analyses were created post-hoc and thus results should be interpreted judiciously.

\section{Results}

\section{Demographic and Characteristics}

Characteristics of the intervention vs. usual care (UC) groups was reported previously [10, 11]. In brief, 243 patients were screened for study participation and 168 (69\%) were randomized to either the SCT intervention $(\mathrm{N}=85)$ or to usual care $(\mathrm{N}=83)$. Of these 168 over 12 months, 10 participants did not want to complete further questionnaire packets, 2 developed terminal cancer and decided not to continue, 3 died from heart failure, 1 due to renal failure and 1 due to valvular heart disease, and 1 participant withdrew. Complete data were available on 150 of 168 (89\%) at all time points over 12 months. There were no significant differences in baseline characteristics and outcomes for those who did vs did not complete all data collection. With two exceptions, there were no statistically significant differences between the intervention groups on baseline characteristics. Specifically, participants in the intervention group were more likely to live with a significant other or spouse $(p=0.01)$ and to have suffered a myocardial infarction $(p=0.05)$ prior to receiving the ICD.

Demographic and clinical characteristics between the SCA group $(\mathrm{N}=65)$ vs the other arrhythmia group $(\mathrm{N}=103)$ were examined post-randomization and are reported in Table 1 . There were no statistically significant differences in baseline demographics between SCA and the other ICD arrhythmia group. The other ICD indication group included patients who received an ICD for VT or VF that was inducible on EPS testing (63\%), sustained VT with syncope or pre-syncope (16\%), unmonitored syncope with documented VT (13\%), or VT lasting $>30$ seconds $(9 \%)$. 
Table 1

Demographic and Clinical Characteristics

\begin{tabular}{|c|c|c|c|}
\hline \multirow[t]{2}{*}{ Variables } & $\begin{array}{l}\text { Sudden Cardiac } \\
\text { Arrest }\end{array}$ & $\begin{array}{l}\text { Other Cardiac } \\
\text { Arrhythmia }\end{array}$ & \multirow[t]{2}{*}{ p-value } \\
\hline & $N=65$ & $N=103$ & \\
\hline \multicolumn{4}{|l|}{ Sex } \\
\hline Male & $48(74 \%)$ & $81(79 \%)$ & \multirow[t]{2}{*}{0.47} \\
\hline Female & $17(26 \%)$ & $22(21 \%)$ & \\
\hline Age (mean years) & $63.5 \pm 12.2$ & $64.4 \pm 12.4$ & 0.64 \\
\hline $\mathrm{BMI}($ mean kg/m²) & $27.6 \pm 5.6$ & $28.8 \pm 6.7$ & 0.24 \\
\hline Charlson Co-morbidity Index & $4.0 \pm 2.3$ & $4.6 \pm 2.4$ & 0.11 \\
\hline LVEF\% & $34.8 \pm 15.3$ & $33.1 \pm 12.6$ & 0.47 \\
\hline \multicolumn{4}{|l|}{ Race } \\
\hline Caucasian & $59(91 \%)$ & $91(88 \%)$ & \multirow[t]{5}{*}{0.70} \\
\hline American Indian/Alaskan & $1(2 \%)$ & $2(2 \%)$ & \\
\hline Asian/Pacific Islander & $2(3 \%)$ & $2(2 \%)$ & \\
\hline Black/African American & $3(5 \%)$ & $4(4 \%)$ & \\
\hline Mixed race/Other & $0(0 \%)$ & $3(3 \%)$ & \\
\hline \multicolumn{4}{|l|}{ Education } \\
\hline Some High School or Less & $8(12 \%)$ & $13(13 \%)$ & \multirow[t]{7}{*}{0.64} \\
\hline Graduated High School & $11(17 \%)$ & $27(26 \%)$ & \\
\hline Some College & $15(23 \%)$ & $25(24 \%)$ & \\
\hline Completed Vocational Program & $2(3 \%)$ & $3(3 \%)$ & \\
\hline Completed 2 Year College & $9(14 \%)$ & $9(9 \%)$ & \\
\hline Graduated 4 Year College & $11(17 \%)$ & $10(10 \%)$ & \\
\hline Graduate Degree & $9(14 \%)$ & $16(16 \%)$ & \\
\hline \multicolumn{4}{|l|}{ Employment Status } \\
\hline Full-time & $13(20 \%)$ & $24(23 \%)$ & \multirow[t]{4}{*}{0.88} \\
\hline Part-time & $8(12 \%)$ & $11(11 \%)$ & \\
\hline Not Employed & $4(6 \%)$ & $4(4 \%)$ & \\
\hline Retired & 33 (51\%) & 55 (53\%) & \\
\hline
\end{tabular}




\begin{tabular}{|llll|}
\hline Variables & $\begin{array}{l}\text { Sudden Cardiac } \\
\text { Arrest }\end{array}$ & $\begin{array}{l}\text { Other Cardiac } \\
\text { Arrhythmia }\end{array}$ & p-value \\
\hline Full-time Housewife & $1(2 \%)$ & $\mathbf{N}=103$ & \\
\hline Disabled & $6(9 \%)$ & $3(3 \%)$ & \\
\hline ICD Reason & & $6(6 \%)$ & \\
\hline VF Sudden Cardiac Arrest & $65(100 \%)$ & $0(0 \%)$ & \\
\hline Sustained VT with Presyncope/Syncope & $0(0 \%)$ & $16(16 \%)$ & \\
\hline Unmonitored Syncope with Documented & $0(0 \%)$ & $13(13 \%)$ & \\
\hline VT & $0(0 \%)$ & $9(9 \%)$ & 0.001 \\
\hline VT $\geq 30$ seconds & $0(0 \%)$ & $65(63 \%)$ & 0.52 \\
\hline VT or VF inducible on EPS & $35(54 \%)$ & $61(59 \%)$ & 0.22 \\
\hline Myocardial Infarction & $17(26 \%)$ & $26(25 \%)$ & 0.26 \\
\hline Diabetes Mellitus & $6(9 \%)$ & $15(15 \%)$ & 0.10 \\
\hline COPD & $28(43 \%)$ & $51(50 \%)$ & 0.48 \\
\hline Heart Failure & $7(11 \%)$ & $20(19 \%)$ & 0.33 \\
\hline CVA & $13(20 \%)$ & $19(18 \%)$ & $1(1 \%)$ \\
\hline Smoker & $2(3 \%)$ & $1(1 \%)$ & \\
\hline Active Alcohol Use & $1(2 \%)$ & & \\
\hline Active Opiate Use & & & \\
\hline
\end{tabular}


Table 2

Outcomes from Hospital Discharge to 12 months by Intervention Condition and ICD Indication Variables SCA Other Arrhythmia

$(\operatorname{mean} \pm \mathrm{SD})$

$($ mean \pm SD)

\& Time Intervention Usual Care Total Intervention Usual Care Total

Physical Function

Symptoms (PCA)

\begin{tabular}{lllllll} 
Discharge & $12.03 \pm 7.69$ & $9.78 \pm 6.07$ & $11.09 \pm 7.10$ & $10.53 \pm 6.83$ & $9.91 \pm 7.42$ & $10.19 \pm 7.13$ \\
\hline $1 \mathrm{mo}$ & $9.94 \pm 7.17$ & $10.63 \pm 8.20$ & $10.22 \pm 7.54$ & $7.87 \pm 6.06$ & $8.91 \pm 7.48$ & $8.42 \pm 6.84$ \\
\hline $3 \mathrm{mo}$ & $9.11 \pm 7.89$ & $8.25 \pm 8.14$ & $8.76 \pm 7.93$ & $7.13 \pm 7.47$ & $8.42 \pm 7.65$ & $7.82 \pm 7.56$ \\
\hline $6 \mathrm{mo}$ & $7.91 \pm 6.00$ & $7.09 \pm 7.99$ & $7.59 \pm 6.80$ & $6.37 \pm 5.65$ & $9.52 \pm 7.57$ & $8.04 \pm 6.88$ \\
\hline $12 \mathrm{mo}$ & $7.03 \pm 6.22$ & $7.23 \pm 8.55$ & $7.11 \pm 7.19$ & $7.39 \pm 6.99$ & $9.58 \pm 8.73$ & $8.57 \pm 8.01$ \\
\hline Total & $9.30 \pm 7.20$ & $8.67 \pm 7.78$ & $9.05 \pm 7.43$ & $7.87 \pm 6.73$ & $9.27 \pm 7.74$ & $8.62 \pm 7.31$
\end{tabular}

\section{General Physical Health (PCS)}

\begin{tabular}{|lllllll} 
Discharge & $32.32 \pm 8.30$ & $35.35 \pm 8.99$ & $33.58 \pm 8.66$ & $33.40 \pm 9.25$ & $36.61 \pm 9.57$ & $35.14 \pm 9.52$ \\
\hline $1 \mathrm{mo}$ & $37.82 \pm 10.36$ & $40.80 \pm 8.15$ & $39.01 \pm 9.58$ & $38.90 \pm 9.84$ & $41.68 \pm 10.84$ & $40.39 \pm 10.43$ \\
\hline $3 \mathrm{mo}$ & $41.30 \pm 10.16$ & $44.68 \pm 9.54$ & $42.68 \pm 9.97$ & $41.67 \pm 10.91$ & $41.16 \pm 11.33$ & $41.40 \pm 11.08$ \\
\hline $6 \mathrm{mo}$ & $42.65 \pm 10.07$ & $45.98 \pm 7.98$ & $43.96 \pm 9.37$ & $42.39 \pm 10.55$ & $42.58 \pm 11.71$ & $42.49 \pm 11.13$ \\
\hline $12 \mathrm{mo}$ & $42.91 \pm 10.11$ & $42.81 \pm 10.72$ & $42.87 \pm 10.26$ & $40.72 \pm 10.71$ & $41.27 \pm 12.25$ & $41.01 \pm 11.52$ \\
\hline Total & $39.19 \pm 10.50$ & $41.67 \pm 9.75$ & $40.19 \pm 10.26$ & $39.38 \pm 10.68$ & $40.60 \pm 11.27$ & $40.04 \pm 11.01$
\end{tabular}

\section{ICD Shocks}

\begin{tabular}{lllllll} 
Discharge & $0.00 \pm 0.00$ & $0.04 \pm 0.19$ & $0.02 \pm 0.12$ & $0.00 \pm 0.00$ & $0.04 \pm 0.19$ & $0.02 \pm 0.14$ \\
\hline $1 \mathrm{mo}$ & $0.03 \pm 0.16$ & $0.28 \pm 0.68$ & $0.13 \pm 0.46$ & $0.22 \pm 0.81$ & $0.50 \pm 1.78$ & $0.37 \pm 1.42$ \\
\hline $3 \mathrm{mo}$ & $0.19 \pm 0.74$ & $0.04 \pm 0.20$ & $0.13 \pm 0.59$ & $0.13 \pm 0.45$ & $0.09 \pm 0.35$ & $0.11 \pm 0.40$ \\
\hline $6 \mathrm{mo}$ & $0.14 \pm 0.55$ & $0.00 \pm 0.00$ & $0.08 \pm 0.43$ & $0.04 \pm 0.21$ & $0.58 \pm 2.56$ & $0.33 \pm 1.88$ \\
\hline $12 \mathrm{mo}$ & $0.55 \pm 1.48$ & $0.13 \pm 0.46$ & $0.38 \pm 1.18$ & $0.20 \pm 0.73$ & $0.79 \pm 1.65$ & $0.52 \pm 1.34$ \\
\hline Total & $0.17 \pm 0.72$ & $0.10 \pm 0.39$ & $0.14 \pm 0.65$ & $0.12 \pm 0.44$ & $0.37 \pm 1.50$ & $0.27 \pm 1.04$
\end{tabular}

\section{Psychological Adjustment}

\section{Mental Health (MCS)}

\begin{tabular}{|lllllll} 
Discharge & $52.17 \pm 9.56$ & $51.19 \pm 9.38$ & $51.76 \pm 9.42$ & $51.53 \pm 9.88$ & $52.67 \pm 9.22$ & $52.15 \pm 9.50$ \\
\hline $1 \mathrm{mo}$ & $52.98 \pm 7.54$ & $51.39 \pm 9.67$ & $52.35 \pm 8.41$ & $53.52 \pm 9.57$ & $52.75 \pm 8.76$ & $53.11 \pm 9.11$
\end{tabular}




\begin{tabular}{|c|c|c|c|c|c|c|}
\hline $3 \mathrm{mo}$ & $54.00 \pm 7.60$ & $53.20 \pm 9.04$ & $53.67 \pm 8.15$ & $54.32 \pm 9.66$ & $52.87 \pm 9.36$ & $53.54 \pm 9.48$ \\
\hline $6 \mathrm{mo}$ & $52.15 \pm 9.11$ & $55.35 \pm 7.63$ & $53.41 \pm 8.63$ & $55.06 \pm 8.66$ & $52.38 \pm 8.85$ & $53.64 \pm 8.82$ \\
\hline $12 \mathrm{mo}$ & $54.30 \pm 7.56$ & $53.65 \pm 8.30$ & $54.03 \pm 7.80$ & $53.93 \pm 9.24$ & $52.71 \pm 9.41$ & $53.27 \pm 9.30$ \\
\hline Total & $53.09 \pm 8.30$ & $52.86 \pm 8.86$ & $53.00 \pm 8.52$ & $53.66 \pm 9.41$ & $52.68 \pm 9.06$ & $53.13 \pm 9.23$ \\
\hline \multicolumn{7}{|l|}{$\begin{array}{l}\text { Anxiety } \\
\text { (STAI) }\end{array}$} \\
\hline Discharge & $36.76 \pm 12.34$ & $36.57 \pm 11.23$ & $36.68 \pm 11.80$ & $35.23 \pm 11.66$ & $31.71 \pm 10.13$ & $33.32 \pm 10.94$ \\
\hline $1 \mathrm{mo}$ & $34.47 \pm 10.52$ & $33.21 \pm 10.81$ & $33.97 \pm 10.56$ & $32.80 \pm 11.08$ & $31.89 \pm 10.15$ & $32.31 \pm 10.55$ \\
\hline $3 \mathrm{mo}$ & $32.86 \pm 12.06$ & $33.63 \pm 11.29$ & $33.17 \pm 11.66$ & $31.02 \pm 11.52$ & $33.00 \pm 11.29$ & $32.08 \pm 11.38$ \\
\hline $6 \mathrm{mo}$ & $33.15 \pm 11.91$ & $30.27 \pm 9.80$ & $32.02 \pm 11.13$ & $30.11 \pm 10.17$ & $32.88 \pm 11.80$ & $31.58 \pm 11.09$ \\
\hline $12 \mathrm{mo}$ & $31.34 \pm 11.84$ & $30.41 \pm 10.53$ & $30.96 \pm 11.23$ & $31.39 \pm 12.03$ & $32.19 \pm 10.46$ & $31.82 \pm 11.15$ \\
\hline Total & $33.82 \pm 11.76$ & $32.99 \pm 10.86$ & $33.48 \pm 11.39$ & $32.13 \pm 11.35$ & $32.33 \pm 10.71$ & $32.24 \pm 11.00$ \\
\hline \multicolumn{7}{|c|}{ Depression (CES-D) } \\
\hline Discharge & $10.37 \pm 7.82$ & $10.85 \pm 7.32$ & $10.57 \pm 7.56$ & $11.81 \pm 10.78$ & $9.66 \pm 7.55$ & $10.64 \pm 9.18$ \\
\hline $1 \mathrm{mo}$ & $9.64 \pm 7.01$ & $10.21 \pm 9.08$ & $9.87 \pm 7.83$ & $8.67 \pm 8.63$ & $8.23 \pm 8.37$ & $8.43 \pm 8.45$ \\
\hline $3 \mathrm{mo}$ & $9.60 \pm 9.50$ & $8.67 \pm 9.14$ & $9.22 \pm 9.29$ & $8.74 \pm 9.24$ & $8.77 \pm 8.78$ & $8.76 \pm 9.95$ \\
\hline $6 \mathrm{mo}$ & $8.00 \pm 8.32$ & $6.73 \pm 7.46$ & $7.50 \pm 7.95$ & $7.28 \pm 7.07$ & $9.50 \pm 9.02$ & $8.46 \pm 8.20$ \\
\hline $12 \mathrm{mo}$ & $7.84 \pm 8.22$ & $9.00 \pm 9.06$ & $8.31 \pm 8.51$ & $8.27 \pm 8.44$ & $9.48 \pm 8.37$ & $8.93 \pm 8.38$ \\
\hline Total & $9.14 \pm 8.17$ & $9.18 \pm 8.41$ & $9.16 \pm 8.25$ & $8.97 \pm 8.98$ & $9.13 \pm 8.37$ & $9.06 \pm 8.65$ \\
\hline \multicolumn{7}{|c|}{ Self-Efficacy } \\
\hline \multicolumn{7}{|c|}{ SCA Self-Efficacy Expectations (SE) } \\
\hline Discharge & $8.04 \pm 1.99$ & $8.28 \pm 1.64$ & $8.14 \pm 1.85$ & $8.47 \pm 1.70$ & $8.98 \pm 1.18$ & $8.75 \pm 1.45$ \\
\hline $1 \mathrm{mo}$ & $8.35 \pm 1.65$ & $8.75 \pm 1.19$ & $8.51 \pm 1.48$ & $8.88 \pm 1.47$ & $8.98 \pm 1.35$ & $8.93 \pm 1.40$ \\
\hline $3 \mathrm{mo}$ & $8.74 \pm 1.49$ & $8.66 \pm 1.56$ & $8.71 \pm 1.50$ & $9.00 \pm 1.32$ & $8.95 \pm 1.44$ & $8.97 \pm 1.38$ \\
\hline $6 \mathrm{mo}$ & $8.76 \pm 1.48$ & $8.65 \pm 1.34$ & $8.72 \pm 1.41$ & $9.12 \pm 1.32$ & $8.94 \pm 1.63$ & $9.03 \pm 1.49$ \\
\hline $12 \mathrm{mo}$ & $8.98 \pm 1.30$ & $8.92 \pm 1.71$ & $8.95 \pm 1.47$ & $9.05 \pm 1.31$ & $9.08 \pm 1.25$ & $9.07 \pm 1.27$ \\
\hline Total & $8.56 \pm 1.63$ & $8.64 \pm 1.49$ & $8.59 \pm 1.57$ & $8.90 \pm 1.44$ & $8.98 \pm 1.37$ & $8.95 \pm 1.40$ \\
\hline \multicolumn{7}{|c|}{ SCA Self-management Behavior (SMB) } \\
\hline Discharge & $7.80 \pm 1.64$ & $7.85 \pm 2.15$ & $7.82 \pm 1.85$ & $7.95 \pm 2.13$ & $8.61 \pm 1.46$ & $8.31 \pm 1.82$ \\
\hline $1 \mathrm{mo}$ & $8.25 \pm 1.76$ & $8.72 \pm 1.09$ & $8.44 \pm 1.54$ & $8.77 \pm 1.52$ & $8.82 \pm 1.37$ & $8.80 \pm 1.44$ \\
\hline $3 \mathrm{mo}$ & $8.78 \pm 1.41$ & $8.49 \pm 1.61$ & $8.66 \pm 1.49$ & $8.91 \pm 1.42$ & $9.03 \pm 1.35$ & $8.98 \pm 1.37$ \\
\hline
\end{tabular}




\begin{tabular}{|c|c|c|c|c|c|c|}
\hline $6 \mathrm{mo}$ & $8.91 \pm 1.15$ & $9.00 \pm 1.43$ & $8.94 \pm 1.26$ & $8.99 \pm 1.38$ & $9.11 \pm 1.21$ & $9.05 \pm 1.29$ \\
\hline $12 \mathrm{mo}$ & $9.04 \pm 1.14$ & $8.89 \pm 1.74$ & $8.98 \pm 1.40$ & $9.05 \pm 1.34$ & $9.09 \pm 1.25$ & $9.07 \pm 1.29$ \\
\hline Total & $8.53 \pm 1.51$ & $8.56 \pm 1.69$ & $8.54 \pm 1.58$ & $8.73 \pm 1.63$ & $8.93 \pm 1.34$ & $8.84 \pm 1.48$ \\
\hline \multicolumn{7}{|c|}{ SCA Outcome Expectations (OE) } \\
\hline Discharge & $4.22 \pm 0.47$ & $4.38 \pm 0.48$ & $4.29 \pm 0.47$ & $4.29 \pm 0.53$ & $4.38 \pm 0.49$ & $4.34 \pm 0.51$ \\
\hline $1 \mathrm{mo}$ & $4.24 \pm 0.58$ & $4.46 \pm 0.50$ & $4.33 \pm 0.56$ & $4.46 \pm 0.47$ & $4.31 \pm 0.46$ & $4.38 \pm 0.47$ \\
\hline $3 \mathrm{mo}$ & $4.34 \pm 0.58$ & $4.51 \pm 0.49$ & $4.41 \pm 0.55$ & $4.44 \pm 0.48$ & $4.34 \pm 0.48$ & $4.39 \pm 0.48$ \\
\hline $6 \mathrm{mo}$ & $4.42 \pm 0.56$ & $4.54 \pm 0.45$ & $4.47 \pm 0.52$ & $4.57 \pm 0.49$ & $4.39 \pm 0.55$ & $4.48 \pm 0.53$ \\
\hline $12 \mathrm{mo}$ & $4.39 \pm 0.63$ & $4.48 \pm 0.46$ & $4.43 \pm 0.57$ & $4.55 \pm 0.44$ & $4.40 \pm 0.44$ & $4.47 \pm 0.44$ \\
\hline Total & $4.32 \pm 0.56$ & $4.47 \pm 0.47$ & $4.38 \pm 0.53$ & $4.46 \pm 0.49$ & $4.37 \pm 0.48$ & $4.41 \pm 0.49$ \\
\hline
\end{tabular}

\section{Differences between the SCA and Other Arrhythmia Groups Over Time}

\section{Physical Function}

Physical function indicators included the patient concerns assessment (PCA), general physical health (PCS), and ICD shocks (Table 3). For PCA symptoms, there was a statistically significant indication $x$ time interaction (Wald $X^{2}=8.38, p=0.04$ ), showing that change over 12 months differed by SCA vs other arrhythmias. Patients in the SCA group compared to the Other Arrhythmias group reported more symptoms over 12 months, except at 12 months, when both groups reported similar symptom levels. There was a significant difference in the number of ICD shocks based on intervention and ICD indication (Wald $X^{2}=7.23, p=0.007$ for 2-way interaction), such that patients in the usual care, Other Arrhythmia group received a higher number of ICD shocks. There was also a significant three-way interaction with intervention group described below. 
Table 3

Summary of Model Effects for Time, Intervention Group, ICD Indication, and Interactions

Wald $\mathrm{X}^{2}$ and $\mathrm{p}$-values for Time, Group, Indication, and Interaction Effects

\begin{tabular}{|c|c|c|c|c|c|c|c|}
\hline Variables & Time & Group & Indication & $\begin{array}{l}\text { Group x } \\
\text { Indication }\end{array}$ & $\begin{array}{l}\text { Group x } \\
\text { Time }\end{array}$ & $\begin{array}{l}\text { Indication } \\
\text { x Time }\end{array}$ & $\begin{array}{l}\text { Group x } \\
\text { Indication } \\
\text { x Time }\end{array}$ \\
\hline
\end{tabular}

\section{Physical}

Function

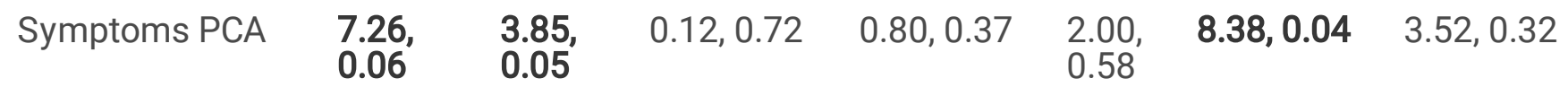

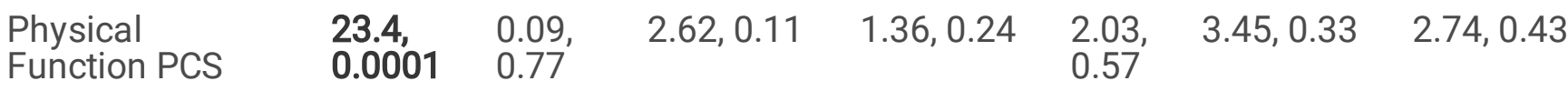

$\begin{array}{llllllll}\text { ICD Shocks } & \mathbf{1 2 . 6 8 ,} & 1.03, & 3.04,0.08 & \begin{array}{l}7.23, \\ 0.007\end{array} & \begin{array}{l}6.52, \\ 0.09\end{array} & 3.29,0.35 & \mathbf{1 0 . 5 8 , 0 . 0 1}\end{array}$

\section{Psychological}

Adjustment

$\begin{array}{llllllll}\begin{array}{l}\text { Mental Health } \\ \text { (MCS) }\end{array} & \begin{array}{l}1.64, \\ 0.65\end{array} & \begin{array}{l}0.69, \\ 0.41\end{array} & 0.07,0.79 & 1.70,0.19 & 1.36,0.72 & 0.68,0.88 & 7.25,0.06 \\ \text { Anxiety (STAI) } & 3.43, & 1.73, & 1.05,0.31 & 2.75,0.10 & 1.74,0.63 & 1.64,0.65 & 2.61,0.46 \\ & 0.33 & 0.19 & & & & & \\ & & & & & & \\ \begin{array}{l}\text { Depression } \\ \text { (CES-D) }\end{array} & 3.94, & 1.23, & 0.04,0.84 & 2.17,0.14 & 1.55,0.67 & 2.24,0.53 & 2.87,0.41 \\ & 0.27 & 0.27 & & & & & \end{array}$

\section{SCA Self-}

Efficacy

$\begin{array}{llllllll}\text { Self-Efficacy } & \mathbf{7 . 8 9}, & 1.96, & 0.05,0.82 & 0.43,0.51 & 4.38, & 2.43,0.49 & 0.87,0.83 \\ \text { Expectations } & \mathbf{0 . 0 5} & 0.16 & & & 0.22 & & \end{array}$

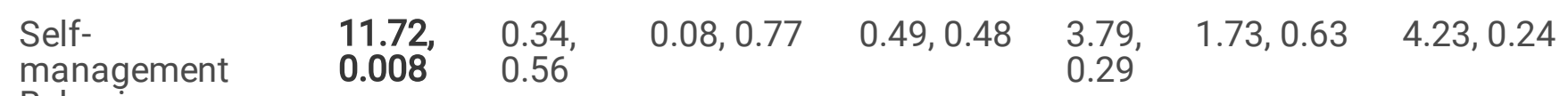

Behavior
Outcome
10.53,
0.75 ,
$0.52,0.47$
6.99 ,
0.008
$0.84, \quad 1.30,0.73$
$0.57,0.90$
Expectations
$\mathbf{0 . 0 1 5} \quad 0.39$
0.84

Group = Intervention vs Usual Care, Indication = sudden cardiac arrest vs other arrhythmia

Bold=significant interactions.

\section{Psychological Adjustment}

Indicators of psychological adjustment included the SF-36 Mental Composite Score (MCS), State Trait Anxiety Inventory (STAI), and Centers for Epidemiology Studies Depression (CES-D) scale. There were no statistically significant differences between SCA and the Other Cardiac Arrhythmia group for outcomes of psychological adjustment (Table 3 ). For the MCS, the general U.S population mean is 50 , with clinically significant change 
reported as 4 mean points [25]. Patients in this study reported mental health perception similar to the U.S. population. Using the STAl, scores of $\geq 30$ reflect moderate anxiety, while scores of $\geq 40$ reflect high anxiety [20]. Participants across all groups reported moderate levels of anxiety throughout the study. On average, study participants did not report clinically significant depression, indicated by CES-D scores $\geq 16$ [21]. Depression scores were the highest at hospital discharge and declined across 12-months.

\section{Self-Efficacy}

Self-efficacy indicators included measures of SCA self-efficacy expectations (SCA-SE), self-efficacy behavior (SCA-B), and SCA outcome expectations (SCA-OE). Across time, there were no significant differences in selfefficacy expectations, self-efficacy behaviors or outcome expectations between the SCA groups or the ICD cardiac arrhythmia group (Table 3).

\section{Differences between the Intervention Groups Over Time}

\section{Physical Function}

PCA symptoms showed differences by group (Wald $X^{2}=3.85, p=0.05$ ), indicating that those in the intervention group reported fewer symptoms compared to usual care over 12 months (Table 3). There were no significant intervention group differences noted by the PCS. Physical health scores ranged in the 30-40s throughout 12 months, generally indicating perceptions of low physical health compared to the general U.S. population. Over time, PCS improved in the intervention group 8-10 mean points, whereas usual care improved an average of 57 mean points.

There was a significant intervention $x$ time $x$ indication interaction for ICD shocks (Wald $X^{2}=10.58, p=0.01$ for 3-way interaction), indicating that over time, the intervention effect was influenced by ICD indication (SCA vs Other Arrhythmias) for secondary prevention (Table 3). Specifically, patients who had a SCA had a similar number of ICD shocks over the 12 months, independent of the intervention group to which they were assigned. Those who received an ICD for other cardiac arrhythmias and participated in usual care, received the highest number of shocks at 1, 6 and 12 months. The intervention was not expected to have a significant impact on the number of ICD shocks.

SCA survivors in the SCT intervention reported greater improvements in physical function over 12-months compared to UC (mean difference from baseline to 12-months): PCA Symptoms: SCA/Intervention -5 points, SCA/UC - 2.55 points; Other arrhythmia/Intervention -3.14 points; Other arrhythmia/UC -0.33 points; PCS: SCA/Intervention +10.59 points, SCA/UC +7.46 points; Other arrhythmia/Intervention +7.32 points; Other ICD/UC +4.66 points. There were no significant differences in those who got ICD shocks, with the exception that the other cardiac arrhythmia group had more reported ICD shocks in the usual care group at 1,6 , and 12 months. ICD Shocks: SCA/Intervention +0.17, SCA/UC +0.10; Other arrhythmia/Intervention +0.12; Other arrhythmia / usual care +0.37 .

\section{Psychological Adjustment}

For general mental health (MCS), the intervention $\mathrm{x}$ time $\mathrm{x}$ indication interaction approached the statistical significance threshold (Wald $X^{2}=7.25, \mathrm{p}=0.06$ ) [Table 3]. With the intervention, mental health gradually improved until 6 months for individuals in the cardiac arrhythmia group, whereas those in usual care showed 
no change. SCA patients in usual care showed sharp improvement from the beginning of the study to 6 months, with a slight reduction in mental health by 12 months. In contrast, the response for SCA patients in the intervention, mental health (MCS) declined from 3 to 6 months, but recovered by 12 months.

The intervention was important in improving depression in SCA participants, while differences by intervention and SCA groups were not observed regarding general mental health and anxiety (mean difference from baseline to 12-months): MCS: SCA/Intervention +2.13 points, SCA/UC +2.46 points; Other arrhythmia /Intervention +2.4 points; Other arrhythmia /usual care +0.04 points; STAl: SCA/Intervention -5.42 points, SCA/UC care -6.16 points; Other arrhythmia/Intervention -3.84 points; Other arrhythmia/usual care +0.48 points; CES-D: SCA/Intervention -2.53 points, SCA/UC care -1.85 points; Other arrhythmia/Intervention -3.54 points; Other arrhythmia/usual care -0.18 points.

\section{Self-Efficacy}

There were no significant differences in self-efficacy expectations or behaviors between the two intervention groups. However, there was a statistically significant Intervention $\mathrm{x}$ indication interaction effect for outcome expectations (Wald $X^{2}=6.99, \mathrm{p}=0.01$ ) [Table 3]. Figure 1 illustrates that for participants in the Other Arrhythmias group, those in the intervention compared to usual care, consistently reported higher outcome expectancies throughout the 12 months. In contrast, for patients who had experienced SCA, those in the intervention compared to usual care, consistently reported lower outcome expectancies.

The SCT intervention played an important role in improving self-efficacy in SCA participants with a higher rate of change over 12-months, but this was small (mean difference from baseline to 12-months): SCA-SE: SCA/Intervention +0.94 points, SCA/UC care +0.64 points; Other arrhythmia/Intervention +0.58 points; Other arrhythmia/UC care +0.1 points; SCB: +1.24 points, SCA/UC care +1.04 points; Other arrhythmia/Intervention +1.1 points; Other arrhythmia/usual care +0.48 points; $S C A-O E$ : SCA/Intervention +0.17 points, SCA/UC care +0.1 points; Other arrhythmia/Intervention +0.26 points; Other arrhythmia/UC +0.02 points. Outcome expectations were higher across 12 months in those in the intervention who did not suffer SCA.

\section{Discussion}

This study indicated that SCA survivors who received an initial ICD, compared to individuals who did not suffer a SCA but had an ICD implanted for a ventricular arrhythmia, had differing trajectories in physical function, psychological adjustment, and self-efficacy outcomes over 12 months while participating in a SCT intervention. Specifically, SCA survivors compared to those with other cardiac arrhythmias had: a) higher numbers of self-reported symptoms regardless of the intervention, $b$ ) non-linear trajectory of recovery in selfreported mental health, with both indication groups ending up a similar mental health at 12 months, c) lower numbers of ICD shocks over 12 months in the SCT intervention compared to those with other arrhythmias, and d) lower outcome expectations over 12 months, but similar levels for both groups at 12 months. This analysis demonstrates that while overall secondary prevention patients benefitted from the SCT intervention that was implemented directly after hospital discharge, the intervention had differing impact on outcomes in SCA survivors compared to the other cardiac arrhythmia group. 
Prior systematic reviews ( $\mathrm{N}=4$ ) of $\mathrm{QOL}$, physical, and psychosocial function following SCA have demonstrated there is significant heterogeneity in survivor characteristics, methodologies and measurement tools used, limiting conclusions that can be made of the impact. Elliott et al [26] conducted a review of 70 studies of patient centered outcomes post-SCA, concluding that QOL after SCA was generally good in 46 studies, neutral in 17 studies, and poor in 7 studies. When survivors were asked to compare current QOL with the pre-SCA QOL, two studies $[27,28]$ noted that almost all judged they were happy with current QOL. Green et al [29] reviewed cognitive function, QOL, and mental health post-SCA, concluding that fatigue is a common long term symptom, some survivors suffer from lack of independence in activities of daily living (ADLs) early in recovery, and mental health concerns are prevalent (61\% experienced anxiety, 45\% depression, 27\% PTSD). Haydon et al [30] reviewed 36 studies, noted that because of differing definitions of QOL, comparisons across studies was difficult. In general QOL post-SCA was good, and did not differ markedly from the general population, ICU patients, or other survivors of cardiac disease. The 2 papers reporting QOL > 15 years post-SCA, demonstrated that QOL was acceptable longer term. This variability in measurement times and instruments has prompted suggestions that a more standard approach to assessment of patient centered outcomes following SCA be adopted [31].

A meta-synthesis of seven qualitative reports exploring the experiences of survival after SCA [32] identified five themes common post-resuscitation from baseline hospital discharge up to 15 years. Themes included: 1) multitude of contrasting feelings such that survival creates the full range of feelings from fear, loneliness, and vulnerability, to gratefulness and joy; 2) disruption in the continuum of time or the realization that the natural course of life was changed by the SCA, this threat of life exposes one's mortality; 3) new reality and psychological challenges with a redefinition of attitude toward life, disbelief, surprise, and not taking life for granted; 4) changed body with new limitations or the inability of the body to function as usual, including cognitive problems and inability to do everyday activities; and 5) confrontation with death or the finiteness of life, with realization of how fortunate survivors were to be alive. Conclusions also noted heterogeneity in sample characteristics and measurement times, noting the absence of specialized tools that capture the survivor experience after SCA.

In this study, those who had a SCA reported higher symptoms and fewer ICD shocks across 12-months. Higher levels of physical symptoms may be expected in SCA participants given they are recovering from resuscitation, ICU stays, and prolonged hospitalization. In addition, SCA participants reported lower physical health in the first month after the ICD implantation. However, from 3 to 12 months, SCA survivors reported improved physical health that became superior to the participants receiving an ICD for other cardiac arrhythmias.

Following hospital discharge, SCA survivors may experience a number of significant physical health impairments including musculoskeletal (MSK), neurological, and cognitive issues lasting weeks to months. Muscle weakness (7-17\%), fatigue (50\%), chest wall pain from CPR and procedures (4-66\%), and speech or swallowing difficulty (0-11\%) can be present for 3-12 months [1]. Physical and occupational therapy, exercise interventions, and other specific therapies have been shown to improve physical function. Education to patients and families about expectations post-arrest can improve adaptation to physical impairments [33]. 
Neurological and cognitive sequelae after SCA occur because of interrupted and inadequate blood flow to the brain, resulting in a cascade of post-arrest changes that impact physical function, including seizures, movement disorders, ataxia, loss of short term memory, and reductions in attention and executive function [1]. These impairments may affect $24-44 \%$ of SCA survivors initially and over time. Medications, physical and occupational therapy, sensory feedback, memory aids, metacognitive training, speech-language therapy, and enlisting the assistance from caregivers has been shown to improve these impairments. Neurological and cognitive impairments are linked to lower QOL and inability to return to work $[34,35]$.

Psychological adjustment was lower in SCA survivors in overall mental health, anxiety, and depression across the first 6 months. However, at 12 months, SCA survivors reached similar levels of psychological adjustment to those who did not have a SCA in all three psychological adjustment outcomes. The psychological responses following out-of-hospital SCA has been an important area of investigation for many years, publications pre-date the use of the ICD. In the years previous to the use of the ICD, three authors [36-38] using open-ended or psychiatric interviews, noted that survivors of cardiac arrest experienced elevated anxiety, depression, fear of being alone, loss of autonomy, and lower social functioning. As well, Dougherty [39] noted that anxiety, depression, anger, and stress was elevated in SCA survivors who received an ICD shock compared to those who had no ICD shock over the 1st year following resuscitation.

In the longest follow-up reported in SCA survivors (up to 8 years) [42] noted that PTSD was present in 27\%, with these individuals also reporting lower quality of life, self-care, and more pain and depressed mood. Those who were younger reported higher levels of PTSD. Kamphius [43] followed SCA survivors over 12 months noting that significant anxiety was present in $61 \%$ at hospital discharge that was reduced to $49 \%$ over 12 months, depression was present in $37 \%$ and was not reduced at 12 months of follow-up. Comparing SCA survivors who received therapeutic hypothermia to those with STEMI after 6 months [44], SCA survivors had more frequent anxiety ( $24 \%$ vs $13 \%$ ) and more depression (19\% vs $8 \%$ ). Anxiety and depression levels were more common in women and those who were younger. Factors related to more psychological distress include receiving ICD shocks, younger age, being female, and having more disease burden. Interventions used to address psychological recovery after SCA have included medications, cognitive behavioral therapy, selfmanagement interventions, and psychotherapy [1].

Dimensions of self-efficacy had a similar trajectory independent of having a SCA. Self-confidence to care for oneself, self-management behaviors, and outcome expectations were similarly stable in all groups over time. Self-efficacy is the belief about one's capabilities to exert control or self-confidence to perform a behavior, and is thought to be the most powerful causal determinant of motivation and performance [14]. Interventions to improve physical and psychological adjustment in post-ICD recovery have been developed, but self-efficacy, a potential mechanism of intervention effectiveness, has not often been studied as a mediator or an outcome of interventions. Evidence from RCTs that have tested the use of the SCT interventions demonstrate outcome benefits in a variety of chronic and cardiac conditions [45-48]. In our intervention research, we have demonstrated the important role of self-efficacy in impacting physical and psychological outcomes after an $\operatorname{ICD}[10,11,49]$. In SCA survivors, strengthening elements of the intervention to impact self-efficacy and outcome expectations need to be considered. 
Importantly, while medical care for SCA has evolved over the last decade, little has changed in the overall assessment, rehabilitation, and chronic illness management of the longer term physical and psychological effects of resuscitation from SCA. Our SCT intervention was one of the first studies to implement a homebased telephone nursing intervention to impact health outcomes after ICD implant [10,11]. At the time the study was conducted, little was known about the trajectory of recovery in physical and mental of SCA survivors compared to all others who had a secondary indication for ICD implant. By determining the specific response to the SCT intervention in SCA survivors, future clinical care can be designed to address these unique needs in the SCA survivors, including recognition and management of symptoms, provision of more assessment and management of mental health issues, and increasing confidence that implementing self-care will improve one's overall health.

\section{Strengths/Limitations}

This RCT was conducted before the approval of ICD implantation for primary prevention of SCA, thus all individuals receiving an ICD did so for secondary prevention. There was a relatively large number of SCA survivors who participated in the study over a short period of time. Data were collected prospectively so that changes and trends in outcomes could be described and reported. The SCT intervention was carried out with high fidelity, and there were few drop-outs in the study over 12 months. Reliable and valid measures were used to represent each outcome of interest. The limitations of the analysis include the data were collected prior to the widespread use of therapeutic hypothermia post-resuscitation, thus the levels of the outcomes may not be similar to SCA survivors today. As well, the study was not powered to find statistically significant differences between SCA and other cardiac arrhythmia groups, thus results are considered exploratory and descriptive.

\section{Conclusions}

Must of clinical care post-SCA is unchanged over the last several decades. SCA survivors had higher physical symptoms, lower number of ICD shocks, lower levels of mental health and outcome expectations over 12 months despite a SCT intervention. Future research should address the unique and special needs of SCA survivors in the early post-resuscitation period, as well as the longer term physical and mental health sequelae of SCA survival. Comprehensive assessment of physical and mental health at the time of hospital discharge should be instituted, so that important issues can be discovered and addressed to improve overall QOL in the aftermath of SCA. Following assessment, symptom management, psychological support, and rehabilitation interventions should be made available during the first year after SCA to impact recovery.

\section{List Of Abbreviations}

ANOVA-analysis of variance

CES-D-Centers for Epidemiologic Studies- Depression,

EPS-electrophysiological study

GEE-generalized estimating equations 
ICD-implantable cardioverter defibrillator

IRB-institutional review board

LVEF-left ventricular ejection fraction

MCS-mental composite score

NTS-nursing telephone support

OE: outcome Expectations

PCA-patient concerns assessment

PCS-physical composite score

QOL-quality of life

SCA-SE- Sudden Cardiac Arrest Self-Efficacy:

SCA-sudden cardiac arrest

SCT-social cognitive theory

SI-structured information

SMB-self-management behaviors

STAl- State Trait Anxiety (STAI),

UC-usual care

VF- ventricular fibrillation

VT- ventricular tachycardia

\section{Declarations}

Ethics approval and consent to participate: The study was reviewed by the University of Washington Institutional Review Board (IRB) before study procedures were taken. All participants provided informed consent. The protocol was performed in accordance with the relevant guidelines and regulations.

Consent for publication: not applicable

Availability of data and materials: The datasets used and/or analyzed during the current study are not publically available because we are not finding a suitable repository, but are available from the corresponding author on request.

Competing interests: The authors have no competing interests to disclose related to the manuscript. 
Funding: This work was supported by the National Institutes of Health [NINR, R01NR04766-01], Dougherty PI.

Authors' contributions:

CMD: conception, design of the work, acquisition of funding, data analysis, interpretation of data, drafted manuscript and substantively revised, approved the submitted version, and agreed both to be personally accountable for the author's own contributions and to ensure that questions related to the accuracy or integrity of any part of the work, even ones in which the author was not personally involved, are appropriately investigated, resolved, and the resolution documented in the literature.

ACSL: interpretation of data, drafted tables and figures, approved the submitted version, and agreed both to be personally accountable for the author's own contributions and to ensure that questions related to the accuracy or integrity of any part of the work, even ones in which the author was not personally involved, are appropriately investigated, resolved, and the resolution documented in the literature.

MMS: interpretation of data, drafted tables and figures, drafted parts of manuscript, approved the submitted version, and agreed both to be personally accountable for the author's own contributions and to ensure that questions related to the accuracy or integrity of any part of the work, even ones in which the author was not personally involved, are appropriately investigated, resolved, and the resolution documented in the literature.

RLB: data analysis, interpretation of data, approved the submitted version, and agreed both to be personally accountable for the author's own contributions and to ensure that questions related to the accuracy or integrity of any part of the work, even ones in which the author was not personally involved, are appropriately investigated, resolved, and the resolution documented in the literature.

KYK: created parts of manuscript and substantively revised, approved the submitted version, and agreed both to be personally accountable for the author's own contributions and to ensure that questions related to the accuracy or integrity of any part of the work, even ones in which the author was not personally involved, are appropriately investigated, resolved, and the resolution documented in the literature.

TZ: created parts of manuscript and substantively revised, approved the submitted version, and agreed both to be personally accountable for the author's own contributions and to ensure that questions related to the accuracy or integrity of any part of the work, even ones in which the author was not personally involved, are appropriately investigated, resolved, and the resolution documented in the literature.

JPA: created parts of manuscript and substantively revised, approved the submitted version, and agreed both to be personally accountable for the author's own contributions and to ensure that questions related to the accuracy or integrity of any part of the work, even ones in which the author was not personally involved, are appropriately investigated, resolved, and the resolution documented in the literature.

EAT: conception, data analysis, interpretation of data, manuscript substantively revised, approved the submitted version, agreed both to be personally accountable for the author's own contributions and to ensure that questions related to the accuracy or integrity of any part of the work, even ones in which the author was not personally involved, are appropriately investigated, resolved, and the resolution documented in the literature.

Page 20/25 
Acknowledgements: none

Disclosures: The authors have no conflicts of interest to disclose.

\section{References}

1. Sawyer KN, Camp-Rogers TR, Kotini-Shah P, Del Rios M, et al. Sudden Cardiac Arrest Survivorship: A scientific statement from the AHA. Circulation, 2020; 141: 00-00. DOI: 10.1161/Cir.00000000000747.

2. Mozaffarian D, Benjamin EJ, Go AS, Arnett DK, Blaha MJ, Cushman M, Das SR, de Ferranti S, Després J-P, Fullerton HJ, et al; on behalf of the American Heart Association Statistics Committee and Stroke Statistics Subcommittee. Heart disease and stroke statistics-2016 update: a re-port from the American Heart Association [published correction appears in Circulation. 2016;133:e599]. Circulation. 2016;133:e38e360.

3. Virani SS, Alonso A, Benjamin EJ et al. Heart disease and stroke statistics-2020 Update: A report from the American Heart Association. Circulation, 2020; 141(9): e139-596.

4. CARES Cardiac Arrest Registry to Enhance Survival. https://mycares.net/. Accessed 8/20/21.

5. Gregoratos G, Abrams J, Epstein AE, et al. ACC/AHA/NASPE2002 guideline update for implantation of cardiac pacemakers antiarrhythmia devices-summary article: a report of the American College of Cardiology/American Heart Association, Task Force on Practice Guidelines (ACC/AHA/NASPE Committee to Update the1998 Pacemaker Guidelines). J Am Coll Cardiol. 2002;40:1703-19.

6. Callaway CW, Donnino MW, Fink EL, Geocadin RG, Golan E, Kern KB, Leary M, Meurer WJ, Peberdy MA, Thompson TM, et al. Part 8: post-cardiac arrest care: 2015 American Heart Association guidelines update for cardiopulmonary resuscitation and emergency cardiovascular care [published correction appears in Circulation. 2017;136:e197]. Circulation. 2015;132(suppl 2):S465-S482.

7. Herrmann C, von zur Mühen F, Schaumann A, Buss U, Kemper K, Wantzen C, Gonska BD. Standardized assessment of psychological well-being and quality-of-life in patients with implanted defibrillators. Pacing Clin Electrophysiol, 1997 Jan;20(1 Pt 1):95-103. doi: 10.1111/j.1540-8159.1997.tb04817.x.

8. Arteaga WJ Windle JR. The quality of life of patients with life-threatening arrhythmias. Arch Int Med, 1995;155: 2086-91.

9. Schron EB, Exner DV, Yao Q, Jenkins LS, Steinberg JS, Cook JR, Kutalek SP, Friedman PL, Bubien RS, Page $\mathrm{RL}$, Powel J. Quality of life in the antiarrhythmics versus implantable defibrillators trial: impact of therapy and influence of adverse symptoms and defibrillator shocks. Circulation, 2002;105(5), 589-594.

10. Dougherty CM, Lewis FM, Thompson ET, Baer, JD, Kim W. Short term efficacy of a telephone intervention after ICD implantation. Pacing and Cardiac Electrophysiology 2004;27: 1594-1602.

11. Dougherty CM, Thompson EA, Lewis FM. Long term outcomes of a nursing intervention after an ICD. Pacing and Cardiac Electrophysiology 2005;28:1157-67.

12. Al-Khatib SM, Stevenson WG, Ackerman MJ, Bryant WJ, Callans DJ, Curtis AB, Deal BJ, Dickfeld T, Field ME, Fonarow GC, Gillis AM, Granger CB, Hammill SC, Hlatky MA, Joglar JA, Kay GN, Matlock DD, Myerburg RJ, Page RL. 2017 AHA/ACC/HRS Guideline for Management of Patients With Ventricular Arrhythmias and the Prevention of Sudden Cardiac Death: A Report of the American College of Cardiology/American 
Heart Association Task Force on Clinical Practice Guidelines and the Heart Rhythm Society. J Am Coll Cardiol. 2018 Oct 2;72(14):e91-e220. doi: 10.1016/j.jacc.2017.10.054.

13. Carpenter CR, Bassett ER, Fischer GM, Shirshekan J, Galvin JE, Morris JC. Four sensitive screening tools to detect cognitive dysfunction in geriatric emergency department patients: Brief Alzheimer's Screen, Short Blessed Test, Ottawa 3DY, and the caregiver-completed AD8. Academic Emergency Medicine: Official Journal of the Society for Academic Emergency Medicine, 2011;18, 374-384.

14. Bandura A. Self-efficacy: Toward a unifying theory of behavioral change. Psych Rev, 1977;84:191-215.

15. Dougherty CM, Benoliel JQ, Bellin C. Domains of nursing intervention after sudden cardiac arrest and automatic internal cardioverter defibrillator implantation. Heart and Lung: The Journal of Critical Care, 2000; 29(2), 79-86.

16. Dougherty CM, Johnson-Crowley NR, Lewis FM, Thompson EA. Theoretical development of nursing interventions for sudden cardiac arrest survivors using social cognitive theory. Advances in Nursing Science, 2001; 20(1), 78-86.

17. Dougherty CM, Pyper GP, Frasz HA. Description of a nursing intervention after ICD implantation. Heart and Lung: The Journal of Acute and Critical Care, 2004;33(3):183-190.

18. Jenkins LS, Dunbar SB, Hawthorne MH. Patient concerns before and the first 9-months after ICD implantation. Circulation 1997;96(8):745

19. Ware J, Kosinski M, Keller SD. A 12-Item Short-Form Health Survey: construction of scales and preliminary tests of reliability and validity. Med Care. 1996;34:220-233. doi: 10.1097/00005650-199603000-00003.

20. Spielberger C, Gorsuch R, Lushene R. Manual for the state-trait anxiety inventory (self-evaluation questionnaire). Palo Alto, CA, Consulting Psychologists, 1970.

21. Radloff LS. The CES-D scale: A self-report of depression scale for re-search in the general population. Appl Psychol Meas 1977; 1:385-401.

22. Dougherty CM, Johnston SK, Thompson EA. Reliability and validity of the self efficacy expectations and outcome expectations after ICD implantation scales. Applied Nursing Research, 2007; 20(3), 116-124. doi:10.1016/j.apnr.2007.04.004

23. Allison PD. Missing Data Techniques for Structural Equation Modeling. Journal of Abnormal Psychology 2003;112(4):545-57.

24. Rothman JK. No adjustments are needed for multiple comparisons. Epidemiology, 1990; 1(1): 43-46.

25. Wyrwich KW, Tierney WM, Babu AN, Kroenke K, Wolinsky FD. A Comparison of Clinically Important Differences in Health-Related Quality of Life for Patients with Chronic Lung Disease, Asthma, or Heart Disease. Health Serv Research, 2005; 40(2): 577-592.

26. Elliott VJ, Rodgers DL, Brett SJ. Systematic review of quality of life and other patient-centered outcomes after cardiac arrest survival. Resuscitation, 2011; 82;247-256.

27. Saner H, Rodriguez, EB, Kummer-Bangerter A, Schüppel R, von Planta, M. Quality of life in long-term survivors of out-of-hospital cardiac arrest. Resuscitation. 2002;53(1):7-13.

28. Harve H, Tiainen M, Poutiainen E, Maunu M, Kajaste S, Roinet RO, Silvast TT. The functional status and perceived quality of life in long-term survivors of out-of-hospital cardiac arrest. Acta Anaesthesiol Scand 2007; 51: 206-209. 
29. Green CR, Botha JA, Tiruvoipati R. Cognitive function, quality of life and mental health in survivors of outof-hospital cardiac arrest: a review. Anaesth Intensive Care, 2015: 43:5, 568-576.

30. Haydon G, van der Riet P, Maguire J. Survivors' quality of life after cardiopulmonary resuscitation: an integrative review of the literature. Scand J Caring Sci; 2017; 31; 6-26.

31. Haywood KL, Pearson N, Morrison LJ, Castrén M, Lilja G, Perkins GD. Assessing health-related quality of life (HRQoL) in survivors of out-of-hospital cardiac arrest: a systematic review of patient-reported outcome measures. Resuscitation. 2018;123:22-37. doi: 10.1016/j.resuscitation.2017.11.065

32. Haydon G, van der Riet P, Inder K. A systematic review and meta-synthesis of the qualitative literature exploring the experiences and quality of life of survivors of a cardiac arrest. European Journal of Cardiovascular Nursing, 2017; 1-9.

33. Walsh TS, Salisbury LG, Merriweather JL, Boyd HA, Griffith DM, Huby G, Kean S, Mackenzie SJ, Krishan A, Lewis SC, et al; RECOVER Investigators. Increased hospital-based physical rehabilitation and information provision after intensive care unit discharge: the RECOVER randomized clinical trial. JAMA Intern Med. 2015;175:901-910. doi: 10.1001/jamainternmed.2015.0822

34. Ørbo M, Aslaksen PM, Larsby K, Schäfer C, Tande PM, Anke A. Alterations in cognitive outcome between 3 and 12 months in survivors of out-of-hospital cardiac arrest. Resuscitation. 2016;105:92-99. doi: 10.1016/j.resuscitation.2016.05.017

35. Steinbusch CVM, van Heugten CM, Rasquin SMC, Verbunt J, Moulaert VRM. Cognitive impairments and subjective cognitive complaints after survival of cardiac arrest: a prospective longitudinal cohort study. Resuscitation. 2017;120:132-137. doi: 10.1016/j.resuscitation.2017.08.007

36. Druss RG \& Kornfeld DS. The survivors of cardiac arrest. JAMA 1967, 201(5): 75-80.

37. Finkelmeier BA, Kenwood NJ, Summers C. Psychological ramifications of survival from sudden cardiac arrest. Critical Care Quarterly, 1984, 7(2): 71-79.

38. Nielsen JR, Gram L, Rasmussen LP, Damsgtuud EM, Dalsgaard M, Richardt C, Beckmann J. Intellectual and Social Function of Patients Surviving Cardiac Arrest outside the Hospital. Acta Med Scand, 1983; 213: 37-39.

39. Sauve MJ, Walker JA, Massa SM, Winkle RA, Scheinman MM. Patterns of cognitive recovery in sudden cardiac arrest survivors: A pilot study. Heart and Lung, 1996, 25(3): 172-181.

40. Kolar JA and Dracup. Psychological adjustment of patients with ventricular arrhythmias. J of CV Nursing, 1990; 4(2): 44-55.

41. Dougherty CM. Psychological reactions and family adjustment in shock versus no shock groups after implantation of internal cardioverter defibrillator. Heart and Lung, 1995;24:281-91.

42. Gamper G, Willeit M, Sterz F, Herkner H, Zoufaly A, Hornik K, Havel C, Laggner AN. Life after death: Posttraumatic stress disorder in survivors of cardiac arrest-Prevalence, associated factors, and the influence of sedation and analgesia. Crit Care Med, 2004; 32:378 -383.

43. Kamphuis HCM, Verhoeven N, de Leeuw R, Derksen R, Hauer R, Winnubst J. ICD: a qualitative study of patient experience the first year after implantation. Journal of Clinical Nursing, 2004, 13, 1008-1016

44. Lilja G, Nilsson G, Nielsen N, Fribergd H, Hassager C, Koopmans M, Martin KA, Mellinghoff MJ, Pelosi P, Wanscher M, Wise MP, Östman I, Cronberg T. Anxiety and depression among out-of-hospital cardiac arrest 
survivors. Resuscitation, 2015; 97:68-75.

45. Baptist AP, Ross JA, Yang Y, Song PX, Clark NM. A randomized controlled trial of a self-regulation intervention for older adults with asthma. J Am Geriatr Soc. 2013; 61: 747-753. doi: 10.1111/jgs.12218 PMID: 23617712

46. Kovar PA, Allegrante JP, MacKenzie CR, Peterson MG, Gutin B, Charlson ME. Supervised fitness walking in patients with osteoarthritis of the knee. A randomized, controlled trial. Ann Intern Med. 1992; 116: 529534. PMID: 1543305

47. Gray CM, Hunt K, Mutrie N, Anderson AS, Treweek S, Wyke S. Weight management for overweight and obese men delivered through professional football clubs: a pilot randomized trial. Int J Behav Nutr Phys Act. 2013; 10: 121-5868-10-121. doi: 10.1186/1479-5868-10-121

48. Furber S, Butler L, Phongsavan P, Mark A, Bauman A. Randomised controlled trial of a pedometer based telephone intervention to increase physical activity among cardiac patients not attending cardiac rehabilitation. Patient Educ Couns. 2010; 80: 212-218. doi: 10.1016/j.pec.2009.11.012 PMID: 20022201

49. Dougherty, C. M., Thompson, E. A., \& Kudenchuk, P. J. (2019). Patient plus partner trial: A randomized controlled trial of 2 interventions to improve outcomes after an initial implantable cardioverterdefibrillator. Heart Rhythm;16(3), 453-459. doi:10.1016/j.hrthm.2018.10.011

\section{Figures}



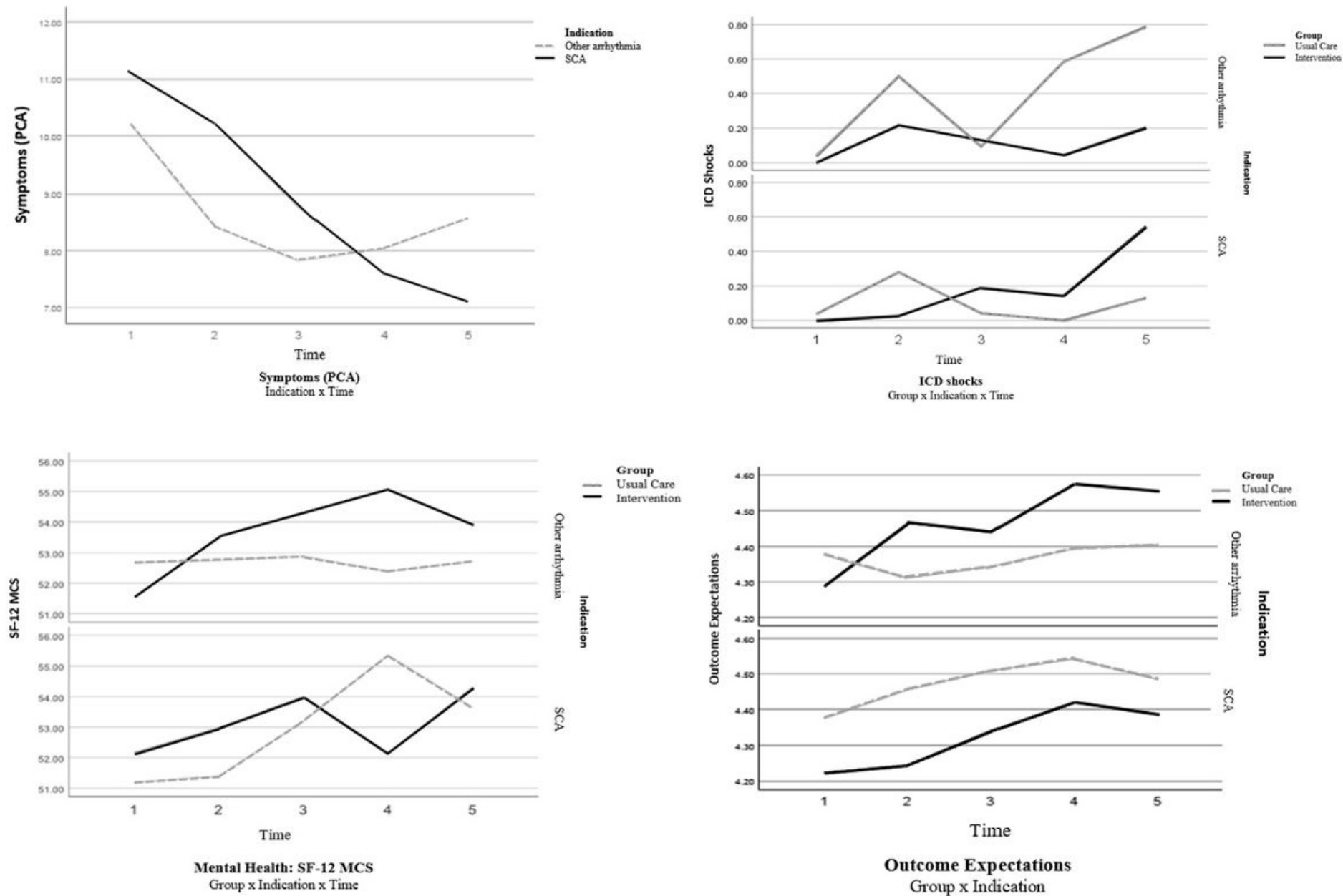

Outcome Expectations Group $\mathrm{x}$ Indication

\section{Figure 1}

Summary of Model Effects for Time, Group, ICD Indication Interactions for PCA, ICD shocks, Mental Health (SF-12 MCS), Outcome Expectations 